\title{
AN ADAPTIVE CAR NUMBER PLATE IMAGE SEGMENTATION USING K-MEANS CLUSTERING
}

\author{
Hana Stefanovicít \\ Radosav Veselinović2, \\ Goran Bjelobaba ${ }^{3}$, \\ Ana Savić ${ }^{4}$
}

'Visoka škola strukovnih studija za IT, Belgrade, Serbia

${ }^{2}$ Ekonomski fakultet,

Belgrade, Serbia

${ }^{3}$ Narodna banka Srbije,

Belgrade, Serbia

${ }^{4}$ Visoka škola elektrotehnike i računarstva stukovnih studija,

Belgrade, Serbia
Correspondence:

Hana Stefanović

e-mail:

hana.stefanovic@its.edu.rs

\begin{abstract}
:
This paper provides an implementation of K-means clustering algorithm in order to segment a car number plate digital image into regions. Some spatial information from a histogram-based windowing process are used, while the user specifies the number of clusters in a dataset and a distance metric to quantify how close two objects in digital image are to each other. Some examples on image segmentation and plate localization for different number of clusters, are illustrated and used in plate characters recognition process.
\end{abstract}

\section{Keywords:}

cluster, data partitions, digital image, K-means algorithm, edge detector.

\section{INTRODUCTION}

Image segmentation is the process of partitioning a digital image into multiple segments or categories presented with sets of pixels [1]. The goal of segmentation is to simplify and/or change the representation of an image into something that is more meaningful and easier to analyze [2]. Image segmentation is typically used to locate objects and some boundaries, like lines, curves, etc., in digital image [3]. In this paper it is used to locate a car number plate region in a digital image.

The result of image segmentation is a set of segments that collectively cover the entire image, or a set of contours extracted from the image. Each of the pixels in a region is similar with respect to some characteristic or computed property, such as color, texture or intensity [4]. Image segmentation is typically used to identify objects or other relevant information in digital images. There are many different ways to perform image segmentation, including: thresholding methods such as Otsu's method, color-based segmentation methods such as K-means clustering, transform methods such as watershed segmentation, and texture methods such as texture filters [5-8]. This paper presents an application of K-means clustering algorithm in order to identify a car number plate region.

An effective approach to perform image segmentation includes different algorithms, tools, and a comprehensive environment for data analysis, visualization, and algorithm development [9]. Some of them, available in MATLAB Image Processing Toolbox ${ }^{\mathrm{TM}}$ are used in this paper 
$[10,11]$, in order to segment a color digital image into regions $[12,13]$. Some spatial information from a histogram-based windowing process are used [1], while a histogram of digital image is produced first by discretisation of the colors in the image into a number of bins, and counting the number of image pixels in each bin $[5,9]$. A histogram-based analysis gives the number of occurrences of each color, or for each color value the number of pixels with that color is counted.

K-means has long been the workhorse for metric data. This method is very attractive because of its simplicity and its local-minimum convergence properties [8]. Some of its disadvantages are that it is slow and scales poorly with respect to the time it takes to complete each iteration. Also, the number of clusters, $K$, has to be supplied by the user. With fixed value of $K$ it empirically finds worse local optima than when it can dynamically alter $\mathrm{K}[5,6]$.

The simplest K-means algorithm produces a clustering of the points in the input into $K$ subsets. It partitions the data points into $K$ clusters such that all points in a given subset belong to the some center. The algorithm keeps track of the centroids of the subsets and proceeds in iterations [6]. Before the first iteration the centroids are initialized to random values. The algorithm terminates when the centroid locations stay fixed during an iteration.

A segmentation could be used for object recognition, occlusion boundary estimation within motion or stereo systems, image compression, image editing, or image database look-up $[1,2,10]$. In this paper it is used to locate the license plate regions from vehicle's image [14, 15].

After isolating the plate on the image and after its conversion from RGB layers to gray-scale layer, the contrast and brightness of the image are adjusted. A Median filter is used to reduce the noise from image [11, 16], while Sobel edge detector $[11,17]$ is applied in order to increase the difference between the letters and the plate backing. Horizontal and vertical image projection are also analysed in order to detect an area of the number plate according to a statistics of the snapshot.

\section{INTENSITY VALUE RANGE OF DIGITAL IMAGE AND K-MEANS CLUSTERING}

An image histogram is a graphical representation of the number of pixels in an image as a function of their intensity [4]. Histograms are made up of bins, each bin representing a certain intensity value range. The histogram is computed by examining all pixels in the image and assigning each to a bin depending on the pixel intensity [2].

In numerical examples presented in this paper the range of pixel values is divided into bins of size $n$ and then quantized, using different number of quantization levels. In such a way the input image will be represented using different number of bins, while coarse representation uses the spatial information from a histogram based windowing process. After that K-means algorithm is used to cluster the coarse image data.

A histogram of digital image is produced first by discretization of the colors in the image into a number of bins, and counting the number of image pixels in each bin. $K$-means algorithm is applied in order to assign each image data object to closest cluster. The objective of $K$-means clustering is to minimize total intra-cluster variance, or, the squared error function $[5,6]$ :

$$
J=\sum_{j=1}^{K} \sum_{i=1}^{n}\left\|x_{i}^{(j)}-c_{j}\right\|^{2}
$$

where $K$ presents the number of clusters, $n$ presents the number of cases, while case $i$ is given with $x_{i}$, and centroid for cluster $j$ is $c_{j}$. Function $\|$.$\| presents the distance$ function, usually Euclidean distance function.

Basic steps in algorithm are: selecting $K$ points as the initial centroids, assigning image objects to their closest cluster center according to the Euclidean distance function, recomputing the centroid of each cluster, and repeating previously described steps until the centroids position do not change. Each cluster in the partition is defined by its member objects and by its centroid, or center.

The centroid for each cluster is the point to which the sum of distances from all objects in that cluster is minimized. In each iteration, the cluster centroids are recomputed, differently for each distance measure, to minimize the sum with respect to the measure that has been specified.

In this paper, the Euclidean distance function is used. Improved K-means algorithm, called k-means++, which includes some specific methods for choosing the initial values for the classical the K-means clustering algorithm $[12,13]$, is not applied in this paper. 


\section{SOME EXAMPLES ON IMAGE SEGMENTATION}

An original image captured using standard smartphone camera is presented in Fig.1. A 41.3 MP image sensor with Carl Zeiss optics and Xenon flash (Nokia Lumia 1020) is used for all test images given in this paper, while modern ANPR systems uses professional cameras, specifically designed for the task [14].

Original image is illustrated in Fig.1, while the image segmentation and regions for different number of clusters, are illustrated in Fig.2, Fig.3, Fig.4 and Fig.5. K-means algorithm is applied on image data represented by different number of bins, for $K=3, K=4$ and $K=5$, respectively.

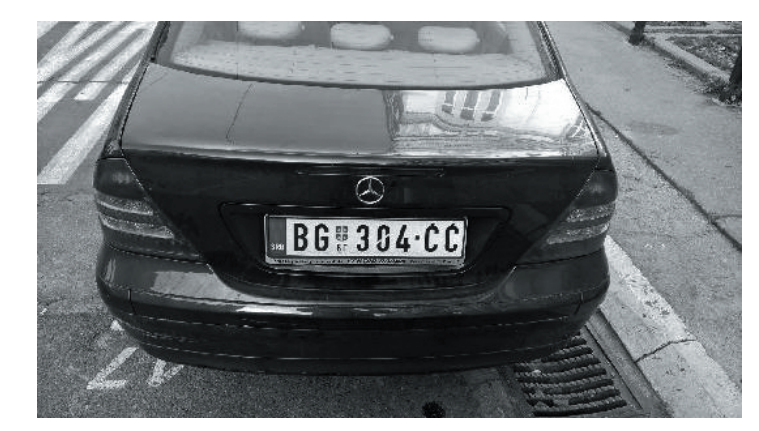

Fig. 1. Original image

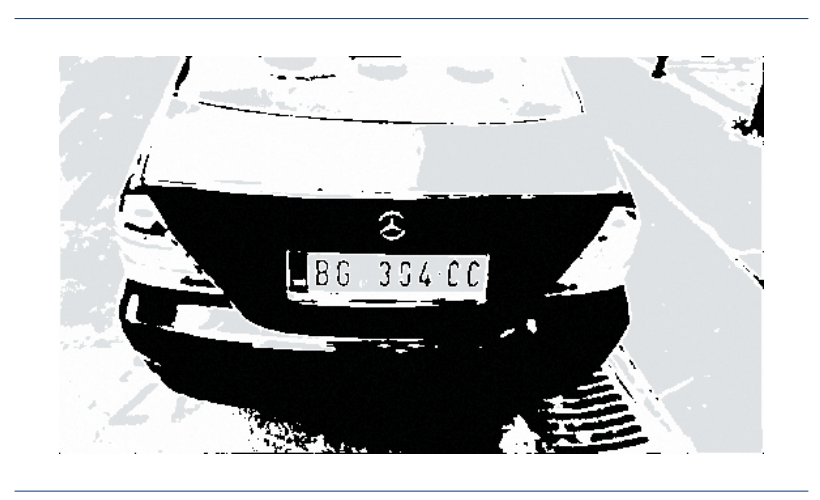

Fig. 2. Regions in image segmented using 3 clusters

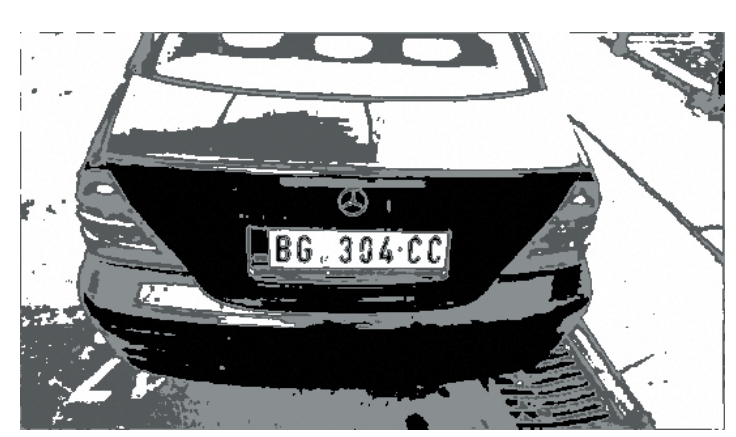

Fig. 3. Regions in image segmented using 4 clusters

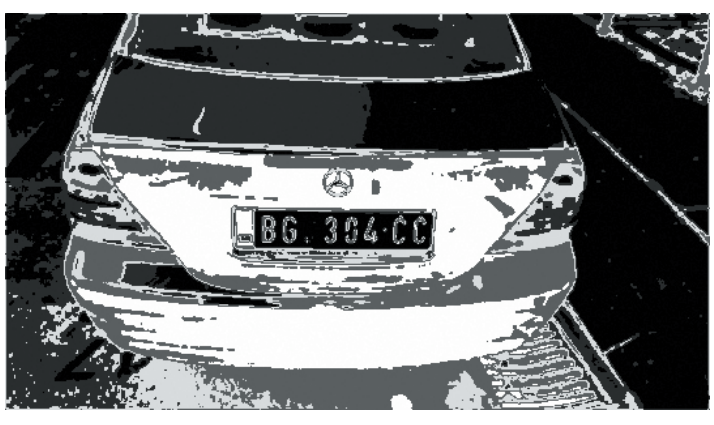

Fig. 4. Regions in image segmented using 5 clusters

The goal of segmentation is to partition digital image into regions each of which has reasonably homogenous visual appearance, as it is illustrated in Figs.2-4. After isolating the plate on the image, the contrast and brightness of the image could be adjusted, and some noisereducing techniques could be used.

\section{SOME METHODS FOR INCREASING THE DIFFERENCE BETWEEN THE LETTERS AND THE PLATE BACKING}

A Median filter is a nonlinear filter, widely used in digital image processing because of its good edge keeping characteristics and reducing impulse noise ability $[11,16]$. It is implemented using MATLAB medfilt function, where each output pixel contains the median value in 3-by-3 neighborhood around the corresponding pixel in the input image. User could also specify some other values of the m-by-n neighborhood around the corresponding pixel in the input image. Results after applying Median filter in order to remove a noise are given in Fig.5.

Sobel detection is an image processing technique for finding the boundaries of objects within images, based on detecting discontinuities in brightness [11, 17]. It is realized using MATLAB edge function, where the gradient of the image is calculated for each pixel position in the image. Results after detecting edges using Sobel detector in order to increase the difference between the letters and the plate backing are given in Fig.6..

The binary gradient mask shows lines of high contrast in the image. These lines do not quite delineate the outline of the object of interest. When compared to the original image, there are some gaps in the lines surrounding the object in the gradient mask. These linear gaps will disappear if the Sobel image is dilated using linear structuring elements, which can be realized using 
strel function in MATLAB [17]. The result after applying basic morphological operations (dilation and erosion) is given in Fig.7 and Fig.8.

Horizontal and vertical image projection are analysed in order to detect an area of the number plate according to a statistics of the snapshot [18]. The vertical projection of the image is a graph, which represents an overall magnitude of the image according to the axis $y$. If we compute the vertical projection of the image after the application of the vertical edge detection filter, the magnitude of certain point represents the occurrence of vertical edges at that point. Then, the vertical projection of so transformed image can be used for a vertical localization of the number plate, as it is illustrated in Fig.9. The horizontal projection represents an overall magnitude of the image mapped to the axis $x$. The characters are segmented according to spaces detected in its horizontal projection. This projection is used to determine horizontal boundaries between characters, while the boundaries correspond to peaks in the graph of the horizontal projection [18], as it is illustreted in Fig.10.

Results after character segmentation are given in Fig.11, while the result after applying free OCR software tool [19, $20]$ is illustrated in Fig.12. Segmentation is based on the fact that the characters of the plate have a certain range of aspect ratio and a certain range of number of pixels.

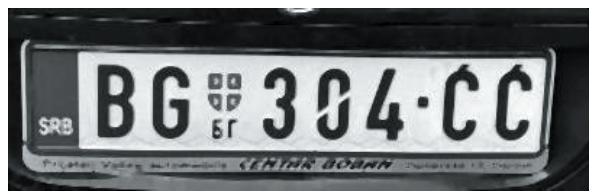

Fig. 5. Result after removing noise using Median filter

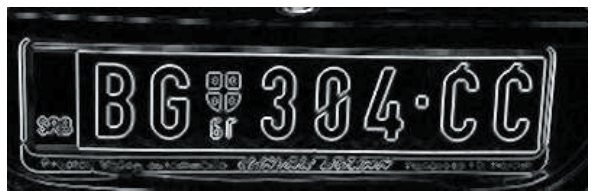

Fig. 6. Result after detecting edges using Sobel operator

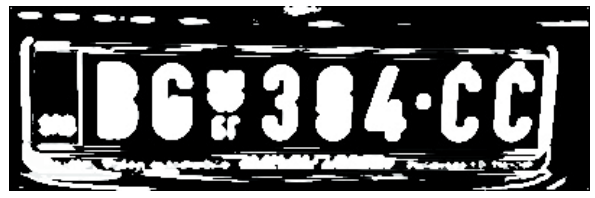

Fig. 7. Result after applying basic morphological operations

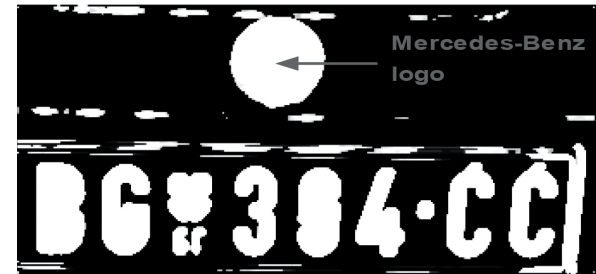

Fig. 8. Detecting and eliminating the Mercedes-Benz logo

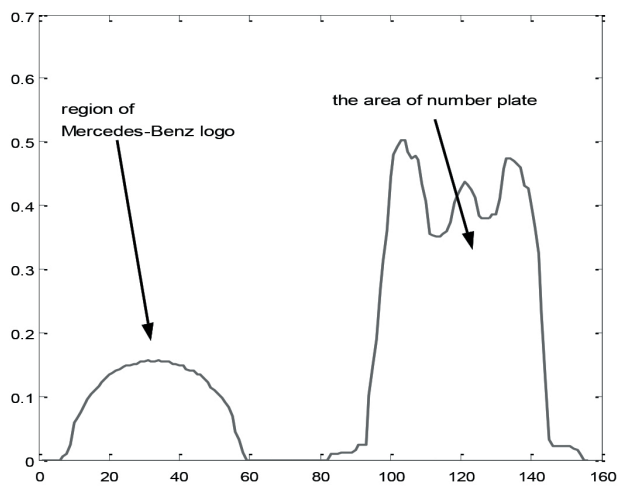

Fig. 9. Vertical image projection

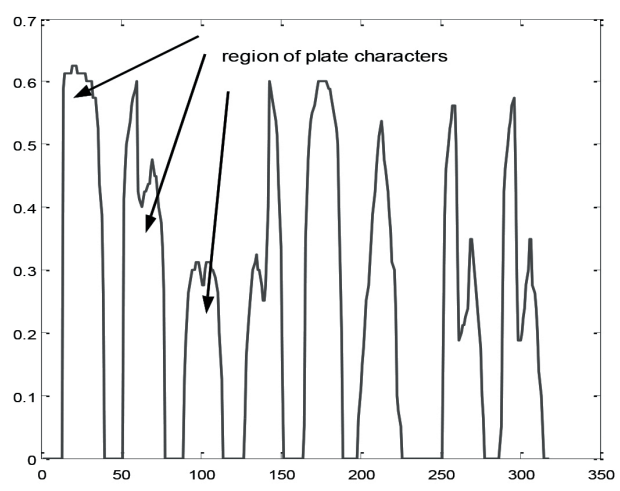

Fig. 10. Horizontal image projection

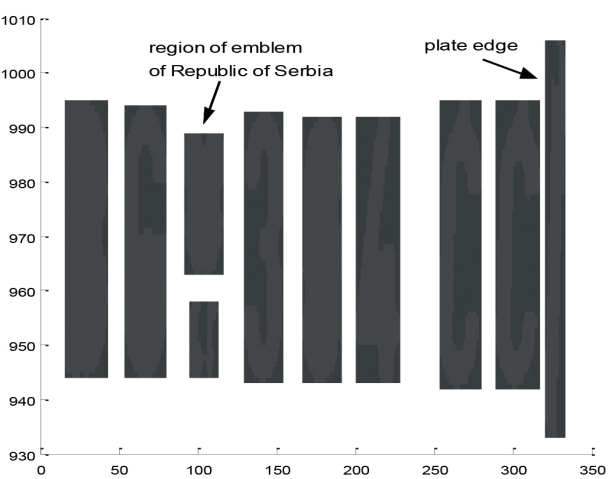

Fig. 11. Characters segmented according to the horizontal projection peaks 


\section{- Ocr Ipload New File}

W Download Output File

BG 304-ĆĆ

Fig. 12. Result after applying some free OCR software tools

After removing the noise, adaptive filter is applied to make the effect of thinning the characters in order to make sure that no two components are merged. Detecting the boundaries between the characters is followed by a horizontal projection, in order to be able to cut them individually. The peaks in horizontal projection correspond to the gaps between the characters, which could be also used in a rejection process, since a true plate has a fixed range of gaps between characters.

\section{CONCLUSION}

This paper contains description and demonstration of simple MATLAB-based image segmentation algorithm, including license plate localization, detecting the boundaries between the characters and optical character recognition, which means translating the segmented characters into text entries. Algorithm is adaptive, so user specifies a number of clusters in image segmentation process, and neighborhood size for median filtering. The algorithm gives satisfactory results in case of slight variation in the same characters due to noise, but it is developed, used and tested in restricted conditions, specific for number plates in Republic of Serbia.

\section{REFERENCES}

[1] W.K. Pratt, Digital Image Processing, Inc. New York, NY, USA., John Wiley \& Sons, 2007.

[2] H.J. Trussell, M.J. Vrhel, Fundamentals of Digital Imaging, UK: Cambridge University Press, 2008.

[3] R.O. Duda, P.E. Hart, Pattern Classification and Scene Analysis, New York: John Wiley\&Sons, 1973.
[4] M. Popovic, Digitalna obrada slike, Beograd: Akademska misao, 2006.

[5] A. Likas, N. Vlassis, J.J. Verbeek, "The global kmeans clustering algorithm", Pattern Recognition, Vol. 36, pp. 451-461, Feb. 2003.

[6] T. Kanungo, D.M. Mount, N.S. Netanyahu, C.D. Piatko, R. Silverman, A.Y. Wu, "An efficient K-means clustering algorithm: analysis and implementation", IEEE Trans. on Pattern Anal and Mach Intell. (TPAMI), Vol. 24, Issue 7, pp. 881-892, Jul. 2002.

[7] P. Arbelaez, M. Maire, C. Fowlkes, J. Malik, "Contour detection and hierarchical image segmentation", IEEE Trans. on Pattern Anal and Mach Intell. (TPAMI), Vol. 33, No. 5, pp. 898-916, May 2011.

[8] R.O. Duda, P.E. Hart, D.G. Stork, Pattern Classification, 2nd Ed., New York: John Wiley\&Sons, 2000.

[9] C. Bishop, Pattern Recognition and Machine Learning, Springer, 2006.

[10] R.C. Gonzalez, R.E. Woods, Digital Image Processing. Upper Saddle River, NJ: Prentice Hall, 2008.

[11] R.C. Gonzalez, R.E. Woods, S.L. Eddins, Digital Image Processing Using MATLAB, Knoxville, TN: Gatesmark Publishing, 2009.

[12] http://www.mathworks.com/help/stats/kmeans. html

[13] D. Arthur, S. Vassilvitskii, "K-means++: The advantages of careful seeding", ACM-SIAM Symp. on Disc. Alg., pp. 1027-1035, 2007.

[14] C. Patel, D. Shah, A. Patel, "Automatic Number Plate Recognition System (ANPR): A Survey”, Int. J. of Computer Applications, vol. 69, no. 9, pp. 2133, 2013.

[15] Z. Lihong, H. Xiangjian, B. Samali, et al.: "Accuracy Enhancement for License Plate Recognition”, IEEE Int. Conf. on Computer and Information Technol.CIT 2010, pp. 511-516, 2010.

[16] https://www.mathworks.com/help/images/ref/medfilt2.html

[17] https://www.mathworks.com/discovery/edge-detection.html

[18] H. Stefanovic, R. Veselinovic, G. Bjelobaba, A. Savic, "Optimizacija algoritmskih resenja za izdvajanje obelezja registarskih tablica u uslovima otezane detekcije“, Info M 64/2017, pp. 33-37, 2017.

[19] http://www.onlineocr.net/

[20] http://www.newocr.com/ 